\title{
Design of Cloud Intelligence Logistics Management System Based on Big Data
}

\author{
Lijuan Ma, Lina Gao \\ Xi’an International University, Xi’an, Shaanxi, 710077
}

Keywords: big data; cloud intelligence; logistics management system

\begin{abstract}
With the increasing integration of the world economy, the traditional logistics distribution mode shows the disadvantages of backward management, low efficiency and unshared information, so it is urgent to have an intelligent logistics management system with high efficiency, convenience, comprehensiveness and decision-making power. In this paper, a cloud intelligence logistics management system based on big data is proposed, which uses big data to optimize decision-making and cloud computing to share computing resources to improve logistics efficiency.
\end{abstract}

\section{Introduction}

With the advancement of globalization, the globalization of resource allocation is getting higher and higher, and the requirements for logistics and distribution are also getting higher and higher. In the massive, high growth rate and diversified information environment, the new processing model is used to achieve greater decision-making power, insight and more optimized processes, which is the top priority for contemporary logistics companies. Therefore, the development of cloud-based smart logistics management system [11] based on big data is the best solution for achieving more efficient and convenient logistics and improving core competitiveness.

\section{Trend Analysis of Logistics Enterprises}

In 2016, Alibaba Group's e-commerce turnover exceeded 3 trillion yuan, and its turnover exceeded Wal-Mart as the world's largest retail platform. This marks the beginning of the circulation revolution and will also fundamentally change the nature of logistics. From the "Double 11" packaging orders, the number of packages from 278 million pieces in 2014 to 467 million pieces in 2015, and then to 2016 has increased to 657 million pieces, service capacity from the initial congestion "explosion", Poor user experience has been transformed into orderly. Compared with 2013 "Double 11", the signing time for 100 million packages is only one-third of the time for signing in 2013. Compared with the "double 11" in 2015, the "double 11" package performance rate in 2016 increased by nearly a quarter [7]. Smart logistics technology is the key to these changes.

Under the pressure of fierce market competition, the profits of low-end services will inevitably become thinner and thinner. According to the different logistics structures, scales, and requirements of various industries, the cost, speed, and service of logistics also vary greatly. This must accelerate. The segmentation of the logistics market will also promote the third-party logistics to provide high-end logistics services, professional solutions and operating modes.

\section{System Function Analysis and Module Design}

Big Data Cloud smart logistics management model is to enter suppliers, inventory, fleet information into the logistics management system, and then upload this information to the big data information sharing platform, so that these resources are shared, so that different logistics companies can achieve information exchange, Resource Sharing. On the basis of resource sharing, the cloud computing platform is reused to provide dedicated decision-making solutions for different customer characteristics, thereby reducing the complexity of logistics management, improving efficiency, solving the drawbacks of poor traditional interoperability, and realizing resource utilization. The increase in the rate has reduced logistics costs. 
The main task of the logistics management system [2] is to provide logistics companies with project management and day-to-day management, and to assist staff in day-to-day affairs management and personnel management, and have achieved the purpose of improving work efficiency, reducing operating costs and personnel costs. Strengthen the competitiveness of enterprises. Through this system, logistics company managers can view logistics-related business information at any time, such as relevant information on consignment notes and customer settlement. Secondly, real-time viewing of fleet conditions, vehicle information, driver information, company outlets, etc., while managing employee information. The system implements data mining [1] and statistical analysis in the registration and storage of large amounts of data information, so as to propose a reasonable logistics plan and realize an efficient and economical operation mode.

The modular design of each function of the system, the main module is divided into five modules of business management, financial management, basic data, SMS center and reporting center, in which business management in order to achieve efficient and efficient way of inquiries, with the most commonly used business as its Sub-module, which includes two parts: consignment note and customer statement; financial management includes only one sub-module of other receipts and payments; the largest one is the basic data module, which includes company network, customer data, vehicle file, and driver. 14 sub-modules, including archives, cover a wide range of information from company to customer, from vehicle to driver, etc. [9]. It is also the main source of big data information. SMS center includes: unsent messages, sent text messages and SMS templates. :

\section{Detailed Design and Implementation of the System}

Smart Logistics [13] integrates the sensor network, the Internet of Things, and the existing Internet to realize visualization, networking, automation, and controllability of logistics management [4] through scientific, accurate, and dynamic management, in order to achieve resource utilization. And raise the level of productivity, thereby creating a more comprehensive social value [3]. How to realize logistics intelligence [6], improve the efficiency of logistics companies, discover new profit opportunities, and need to discover and mine [16] preferences and insights of logistics customers, and this information have the characteristics of large amounts of data, diverse types, and complex structures. Therefore, extracting massive information from big data technology is the best way to solve this problem.

The business management module is the most basic function for real-time business processing and inquiry, and it is also the most commonly used function. Therefore, the module is placed in the most noticeable first item in the navigation bar. The module includes the consignment note and the customer settlement note.

The business management module mainly implements the information of the shipping order, selects the shipping method, confirms the consignment number, date, etc., as well as the information of the customer settlement bill, which facilitates timely checking of the settlement date, amount, etc., and its module interface information.

The basic data module [5] contains comprehensive contents. From the aspect of customer relationship, it includes information from the company's network to customer information. From the perspective of transportation, it includes vehicle files, driver files, transportation methods, vehicle types, etc. From the perspective of company management, it includes information such as employee files, agent files, fee types, cost units, and payment methods.

Considering space limitations, this article only describes the cloud calculator [15] code in the core cloud-brain system program [14] code in the cloud computing as follows: The server receives http data [8] and passes it to the $C$ server. The result is converted into an $\mathrm{xml}$ format and sent back to the client. Server.c receives the data from the java server, calls the mpi program, and returns the result to the java server.

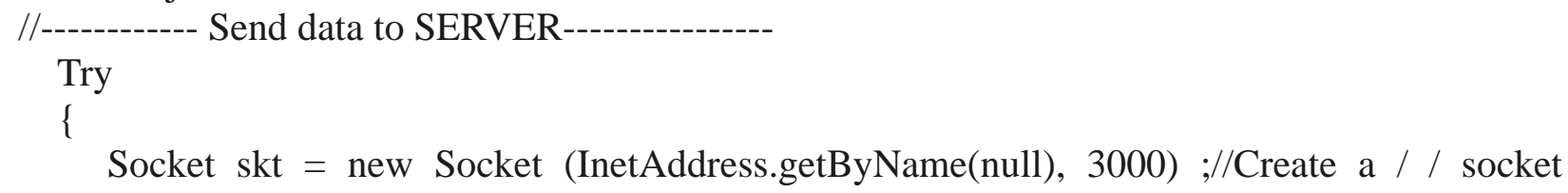


connection, the connection server is the machine, the port number is 3000

System.out.println("Connect to Server "+skt);//Print prompt message

OutputStream ostm=skt.getOutputStream();//Get output stream

If(number==null)

\{

Ostm.write("8". getBytes());

\}

Else

\{

Ostm.write(number.getBytes());//Write to the output stream as the current file encoding

\}

InputStream istm =skt.getInputStream(); //Get input stream

Byte[] info=new byte[512];

Int num=istm.read(info); //Read the input stream into the cache

System.out.println(new String(info,0,num)); //Output the input stream just acquired

Ostm.close();

Istm.close();

Skt.close();

\}

Catch(Exception exp)

\{

\}

exp.printStackTrace();

//---------------- send back to the client

Try

\{

PrintWriter oput=response.getWriter();

Transformer tformer $=$ TransformerFactory.newInstance().newTransformer() ;

//Create an instance to convert

Source scre $=$ new DOMSource (document) $; / /$ XML source file to be converted

StreamResult sresult $=$ new StreamResult(oput) ;

Tformer.transform(scre, sresult) ; //Conversion

Oput.flush();

Oput.close();

\}

Catch (Exception ex)

\{ \}

System.out.println(ex);

\section{Conclusion}

This system introduces big data technology and smart logistics [12], analyzes its characteristics, and links with the status quo of modern logistics, and proposes a design scheme for cloud-based smart logistics management system [10] based on big data. The design of the scheme is first. Determine the framework of the entire management system, analyze its functional implementation according to the module, so as to realize its logistics management system, and finally describe the implementation process of data analysis [17] in detail. Through the actual operation, the interface of the system is simple and intuitive, with detailed information and convenient operation, which can greatly improve the logistics efficiency and optimize the logistics process. 


\section{Acknowledgements}

Xi'an Social Science Fund Project: Research on cloud storage model and distribution strategy based on cloud Logistics(17IN09)

Industrial Science and technology project: Research on IPTV traffic flow evaluation model based on FFTx network(2016GY-114)

\section{References}

[1] J. Dean, S. Ghemawat. Map Reduce simplified data processing on large clusters[C]. In Proceedings of the 6th Symposium on Operating System Design and Implementation (OSDI 2004),2004, pp:137-149.

[2] Wang Xianmei. Research on the theory, method and application of cloud intelligent logistics based on big data [D]. Zhejiang university of science and technology, 2015.

[3] Baidu encyclopedia. Wisdom logistics [EB/OL]. https://baike.baidu.com/item/3105 626. html.

[4] Yan Weihong, Sun Yan, Zhang Yan. Information Oriented: Research On Logistics Management System Based on Multi - Agent [J]. Computer system application.2006, (12):6-9.

[5] Zhong Ming, Su Hongye. B/S - based Logistics Management System and Its Application [J]. Computer application research.2005, (9) :203-205.

[6] Tian Ying. Research and design of logistics positioning management system [J]. Information technology.2012, (12):127-129.

[7] Ali institute, 2017 China smart logistics big data development report full release [EB/OL]. HTTP: // www.sohu.com/a/130842152_384789.html.

[8] Huang Shaochuan. New Design of storage logistics management system [J]. Manufacturing automation. 2012,34(3): 18-21.

[9] $\mathrm{Li} \mathrm{Xi}$. The design and application of chemical resource exchange business logistics management system [J]. Computers and Applied Chemistry. 2013,30(2): 116-120.

[10] Huang Qizhao. Design of Logistics Management Information System Based on Agent Technology [J]. SOFTWARE. 2013,34(8):34-35.

[11] Deng Yanjie, Huang Biqing, Yan Bo. Management pattern and information system of third party logistics enterprises [J]. Computer Integrated Manufacturing Systems.2014,20(5):1220-1227.

[12] ZACHARIAZG, SANDERSNR, NIXNW. The emerging role of the third-party logistics provider(3PL) as an orchestra-tor[J]. Journal of Business Logistics. 2011,32(1):40-54

[13] Chen Ling. Research on the logistics management system of tobacco leaf based on RFID and GIS technology [D]. Xiamen: xiamen university, 2013.

[14] Zhai Yanjie. Design and implementation of mobile learning system based on Hadoop [D]. Henan: henan normal university, 2011.

[15] Yang Yong. Design and implementation of knowledge base based on Lucene [D]. Guangdong: south China university of technology.2011.

[16] Ye Peng, Ma Jun, Wang Wei. Design and implementation of logistics management system based on ARM and RFID [J]. COMPUTER ENGINEERING AND DESIGN. 2013, 34(10):3475-3479

[17] Niklas A-din, Fredrik Stahre. Electronic commerce, marketing channels and logistics platforms a wholesaler perspective[J]. European Journal of Operational Research .2013,14(2), pp:270·-279

[18] Hong, J. Research on fourth-party logistics construction mode of chain enterprises based on cloud computing[C].2011 International Conference on E-Business and E-Government (ICEE),2011:1-4. 